\title{
Étude du projet d'alimentation artificielle de la nappe des sables éocènes en Gironde
}

\author{
par M. Plaud \\ Division Etude des Ressources et des Aménagements \\ Agence Financière de Bassin Adour-Garonne
}

\section{Motivation de l'étude}

L'importance des prélèvements dans la nappe des sables éocènes en Gironde, et notamment dans la région bordelaise, se traduit par un abaissement généralisé de sa surface piézométrique. Ce fait n'est pas nouveau puisque, dès 1955 , le Professeur Schoeller attirait l'attention sur les conséquences à long terme d'une telle évolution et, en particulier, sur le risque d'intrusion d'eau salée dans cet aquifère. Quatre ans plus tard, le décret-loi de 1935 relatif à la protection des eaux souterraines entrait en vigueur dans ce département.

Grâce aux études hydrogéologiques entreprises dès cette époque, la compréhension du système hydraulique fut acquise et un modèle numérique multicouche permit, en 1969 , de simuler l'influence des nouveaux captages préalablement à leur autorisation.

Il faut reconnaître, aujourd'hui, que ces outils technique et législatif se sont avérés inefficaces à enrayer l'évolution de la dépression puisque, depuis une dizaine d'années, elle s'accentue au rythme d'un mètre par an en moyenne, pour atteindre des cotes supérieures à $-5 \mathrm{~m} \mathrm{NGF}$.

Cet abaissement généralisé de la surface piézométrique de la nappe des sables éocènes risquant à terme de compromettre dangereusement son exploitation, l'Agence Financière de Bassin Adour-Garonne a considéré ce problème comme suffisamment préoccupant pour qu'elle examine dès maintenant les moyens permettant d'y remédier.

Parmi les mesures qui pourraient être envisagées, suggérer la réduction autoritaire des prélèvements dans cette nappe est impensable compte tenu des conséquences économiques qui en résulteraient. Ne plus délivrer d'autorisation de prélèvement paraît davantage réalisable; mais l'expérience

* Etudes: Bureau de Recherches Géologiques et Minières; Ecole Nationale Supérieure des Mines de Paris; BURGEAP. montre que, partout ailleurs, l'application de telles mesures conservatoires (dans le bassin Parisien notamment pour la nappe de l'Albien) se révèle insuffisante pour résoudre un tel problème.

Il fallait donc mettre au point une stratégie nouvelle, fondée sur des mesures offensives et réalistes, associées à certaines mesures règlementaires précédemment évoquées, celles-ci étant toutefois rendues plus légères à supporter par les exploitants.

Le problème qui se pose en Gironde est davantage un problème de restructuration du dispositif d'exhaure qu'un problème général de surexploitation caractérisé. En effet, ce dispositif s'est développé, bien évidemment, au gré des besoins, sans tenir compte des caractéristiques hydrodynamiques de l'aquifère, et l'on peut penser que, de le rendre compatible avec ces dernières, contribuerait pour une large part à redresser la situation actuelle.

Dans l'immédiat, il convicndrait:

- de ne plus autoriser de nouveaux captages, ni le remplacement de ceux qui sont vétustes dans la zone la plus déprimée, ceci pourrait ne constituer qu'une mesure transitoire;

- de favoriser l'implantation de captages dans des zones où les conditions hydrogéologiques le permettent;

- de faire cesser tout écoulement inutile sur les forages artésiens abandonnés et sur les forages en exploitation;

- de favoriser, par des aides financières, les adductions d'eau industrielles permettant l'abandon de forages mal implantés au regard de la situation actuelle et (ou) exploités, sans néceessité d'eau de qualité, à des fins industrielles; et enfin, et surtout,

- d'enrayer l'évolution de la surface piézométrique de la nappe et, au minimum, de stabiliser cette dernière à une cote NGF supérieure à zéro par alimentation artificielle.

Il est clair que l'objectif recherché ne peut être atteint que par l'application simultanée de l'ensemble de ces mesu- 
res. $\mathrm{Si}$, en ce qui concerne les premières, il est raisonnable de penser que leurs résultats, bien que non immédiats, seront efficaces, il reste à démontrer que l'alimentation artificielle de la nappe captive en question, située à plus de $200 \mathrm{~m}$ de profondeur en moyenne, est techniquement et économiquement réalisable.

C'est ce que l'Agence Financière de Bassin AdourGaronne a voulu vérifier en adoptant la démarche exposée ci-après :

- choix des zones favorables à l'injection;

- simulations d'hypothèses d'alimentation artificielle sur modèle numérique multicouche;

- étude des coûts.

Seuls les deux premiers points seront abordés ici.

\section{Étude du réservoir}

La figure 1 indique la zone d'étude.

\section{Géologie de l'aquifère}

Cet aquifère a été détini, du point de vue lithostratigraphique, au moyen de dix coupes géologiques, cinq dans le sens NW-SE, c'est-à-dire perpendiculaire au sens de plongement des couiohes, quatre perpendiculaires à la direction précédente et une sensiblement NS.

Les neuf premières constituent une mise à jour des coupes établies en 1967 pour l'« Action concertée Eau du $V^{\mathbf{e}}$ Plan ». La dernière tient compte des forages récents exécutés, pour le compte de la Communauté Urbaine de Bordeaux, au sud de l'agglomération.

La figure 2 indique les limites des faciès du réservoir, la figure 3 représente une coupe schématique SW-NE.

L'examen des coupes lithologiques fait apparaître un aquifère sableux, épais de $70 \mathrm{~m}$ environ, qui passe, à l'ouest, à des calcaires dolomitiques et calcaires sableux. Il faut noter également le passage progressif, vers l'ouest, de ces calcaires à des niveaux de marnes et d'argile.

Dans l'ensemble, ces coupes montrent que l'aquifère est loin d'être homogène sur toute son étendue et sur toute son épaisseur et qu'il est interconnecté localement avec l'éocène inférieur et avec l'éocène supérieur.

\section{Hydrogéologie de l'aquifère}

\section{Infrastructure d'exhaure}

On peut dénombrer 287 ouvrages captant la nappe des sables éocènes en Gironde, dont certains datent de la fin du siècle dernier. Par suite des abandons ou de la création de nouveaux forages, 190 sollicitent actuellement cet aquifère.

Leur répartition et leur équipement est indiqué sur la figure 4; nous pouvons noter leur densité le long du cours inférieur de la Garonne.

Il était intéressant de connaître leurs caractéristiques, afin de pouvoir éventuellement choisir l'un d'entre eux pour effectuer un essai d'injection in situ dans une phase ultérieure.

\section{Caractéristiques hydrauliques}

\section{- Transmissivité}

Les valeurs de la transmissivité exprimées en $\mathrm{m}^{2} / \mathrm{h}$ ont, soit été calculées en régime transitoire, soit déduites du débit spécifique, soit encore évaluées en fonction de la lithologie. Ces valeurs, allant de moins de 1 à 150 , concernent, en effet, l'ensemble de l'aquifère capté quels que soient les faciès.

Les zones les plus favorables sont axées sur les cours inférieurs de la Garonne et de la Dordogne et les courbes d'isotransmissivité ont une orientation générale qui s'apparente aux limites des principaux faciès lithologiques.

\section{- Coefficient d'emmagasinement.}

Une vingtaine de valeurs calculées en régime transitoire constituent l'essentiel de nos connaissances. Le coefficient d'emmagasinement varie de $4,4 \times 10^{-3}$ à $1,7 \times 10^{-5}$, la médiane étant proche de $2 \times 10^{-4}$.

\section{- Débit spécifique.}

Les valeurs varient de 1 à $35 \mathrm{~m}^{3} / \mathrm{h} / \mathrm{m}$ et leur répartition est conforme, comme il se doit, à celle dos transmissivités.

\section{- Température de l'eau.}

Les mesures, étalées dans le temps, ont été effectuées dans des conditions très différentes d'un ouvrage à l'autre. D'une façon générale, on peut remarquer tout naturellement un accroissement de la température avec la profondeur de l'aquifère sans anomalies notables. Elles varient de $17^{\circ}$ à $29,2^{\circ}$.

\section{Piézométrie}

Un historique de la piézométrie a été retracé au travers de quatre cartes en hydroisohypses.

Compte tenu de l'ancienneté des mesures, ces cartes piézométriques passent de l'état figuratif à l'état représentatif entre 1880 et 1972 .

L'examen de la figure 6 (1880-1890) incite à admettre la préexistence d'une dépression axée sur la Garonne et la Dordogne. Remarquons que la courbe hydroisohypse, $+15 \mathrm{NGF}$, passe alors au droit de Bordeaux.

Entre $1880-1890$ et $1940-1950$, on constate une dépression de 7 à $8 \mathrm{~m}$ au niveau de Bordeaux et, en 1965, celle-ci atteint une dizaine de mètres avec une extension de $35 \mathrm{~km}$ dans sa plus grande dimension.

En 1972 (figure 7), on note un glissement de la dépression piézométrique vers le sud et l'apparition de l'hydroisohypse $-5 \mathrm{NGF}$ avec des pointes à -7 et $-9 \mathrm{NGF}$. Cette dernière, de forme elliptique, s'étend sur $25 \mathrm{~km}$ de longueur et sur $10 \mathrm{~km}$ de largeur.

Quatre courbes, issues de limnigrammes enregistrés sur quatre forages situés dans des secteurs différents (figure 8), montrent l'ampleur de l'abaissement annuel du niveau piézométrique ( 1 à $1,30 \mathrm{~m}$ ), dû à l'accroissement des prélèvements. 


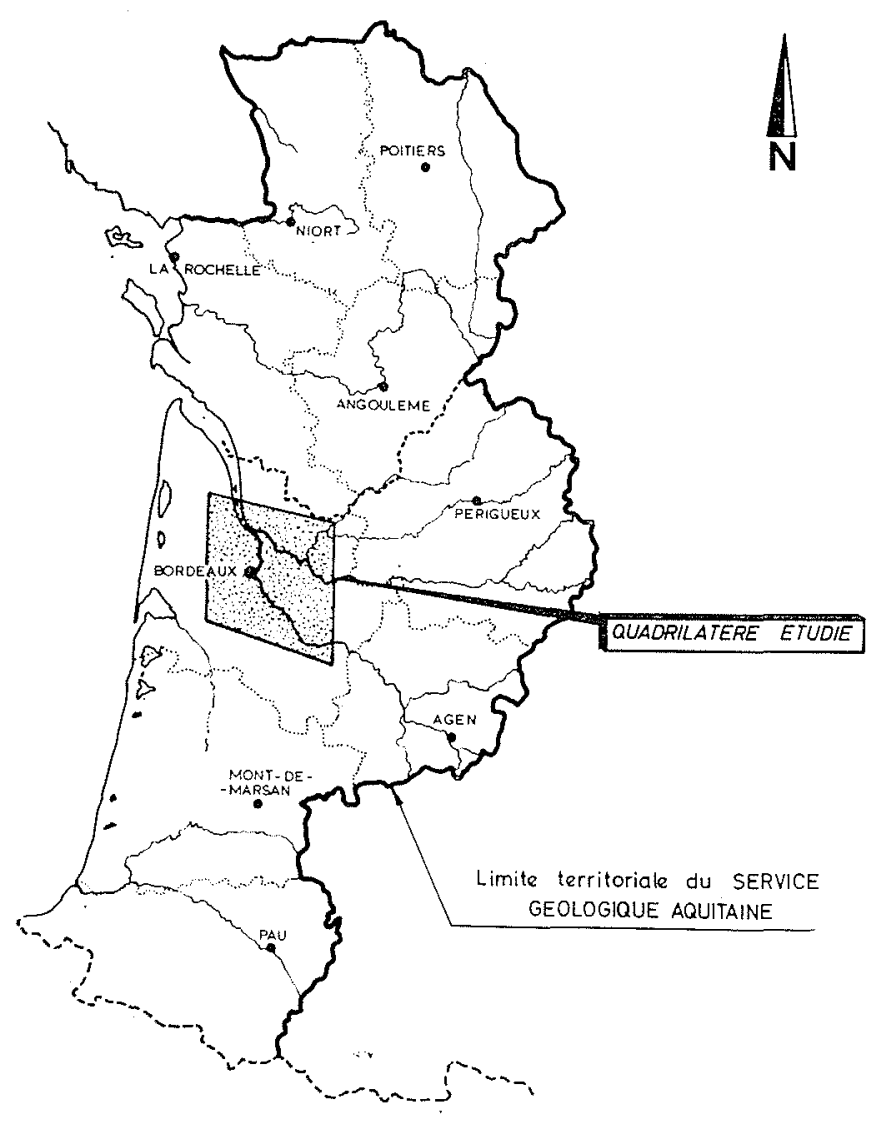

1/ Situation de l'étude.

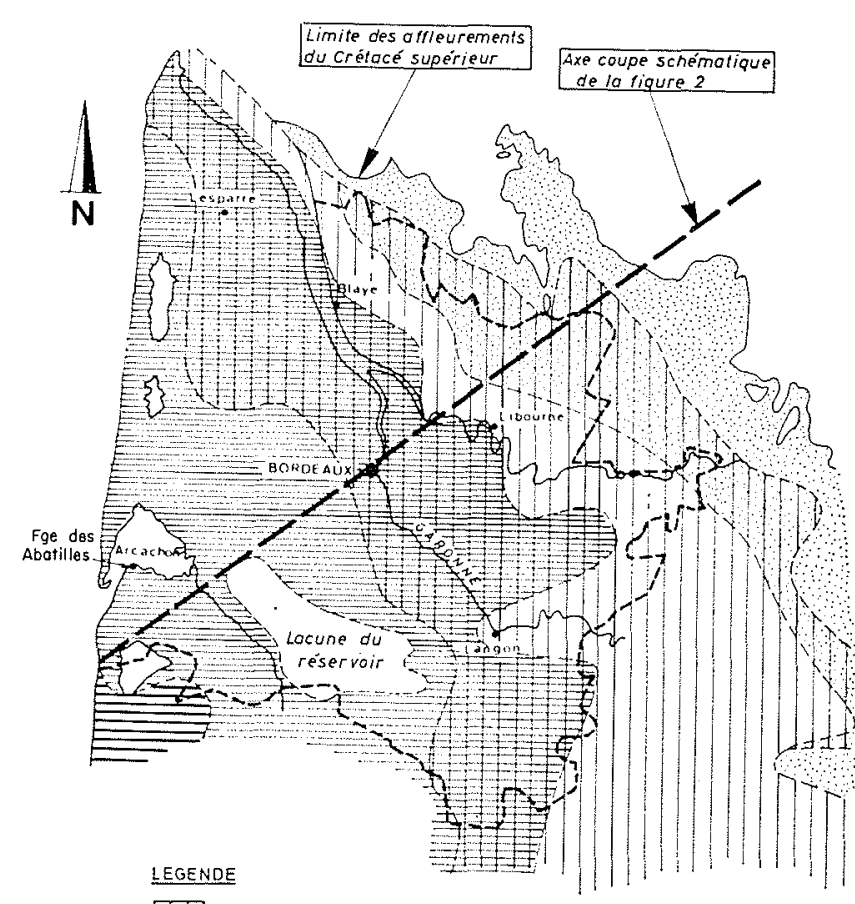

$\prod$ sabies grossiers

3 complexe colcaréo-marneux

Echelle $0 \quad 10 \quad 20 \mathrm{~km}$

ID Complexe de calcaires, grès et sables à couches

Complexe sobto-argileux ( Sables ou Périgord et Sidérolithique)

[D] Sobles argileux superposés aux sables grossiers.

Argiles et mornes

2/ Limite des faciès du réservoir.

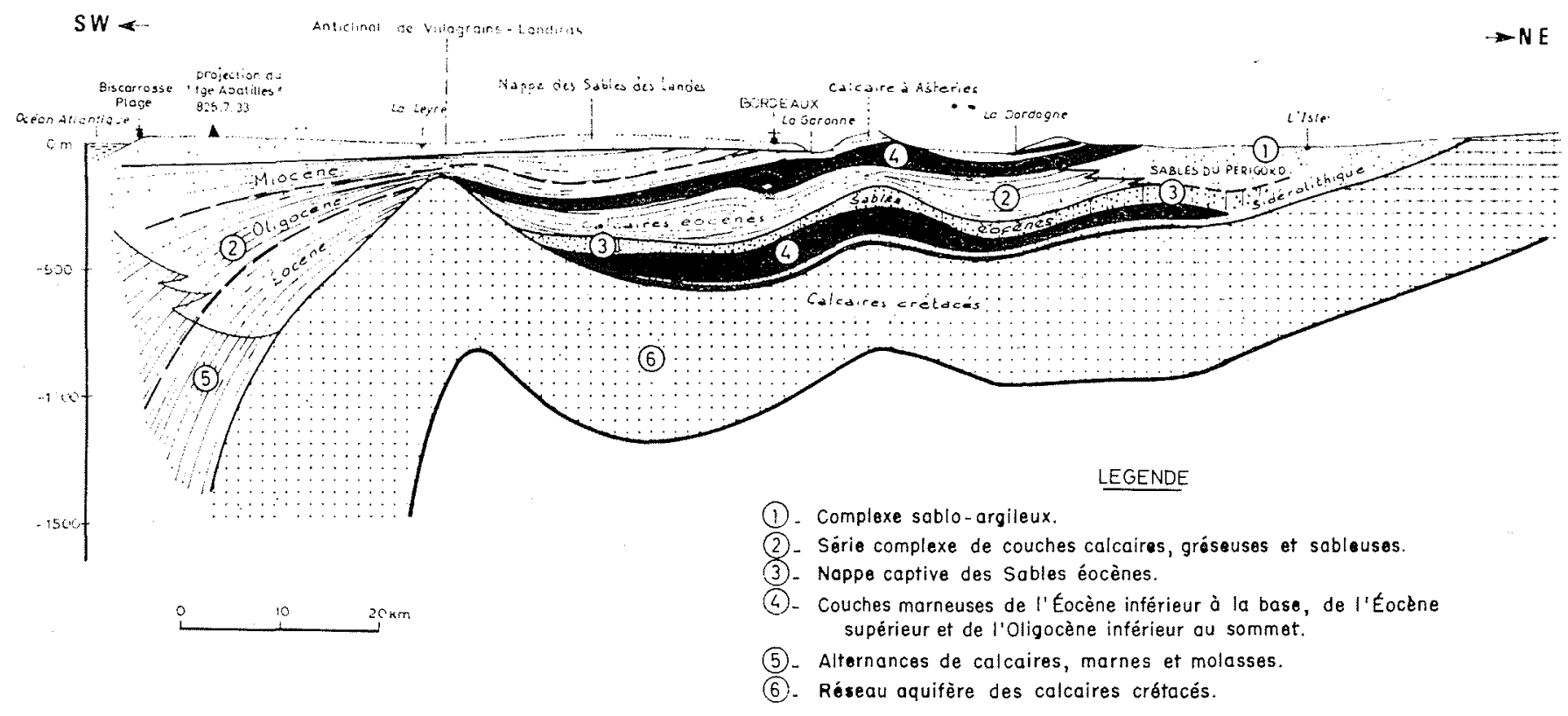

3/ Coupe géologique schématique SO-NE. 


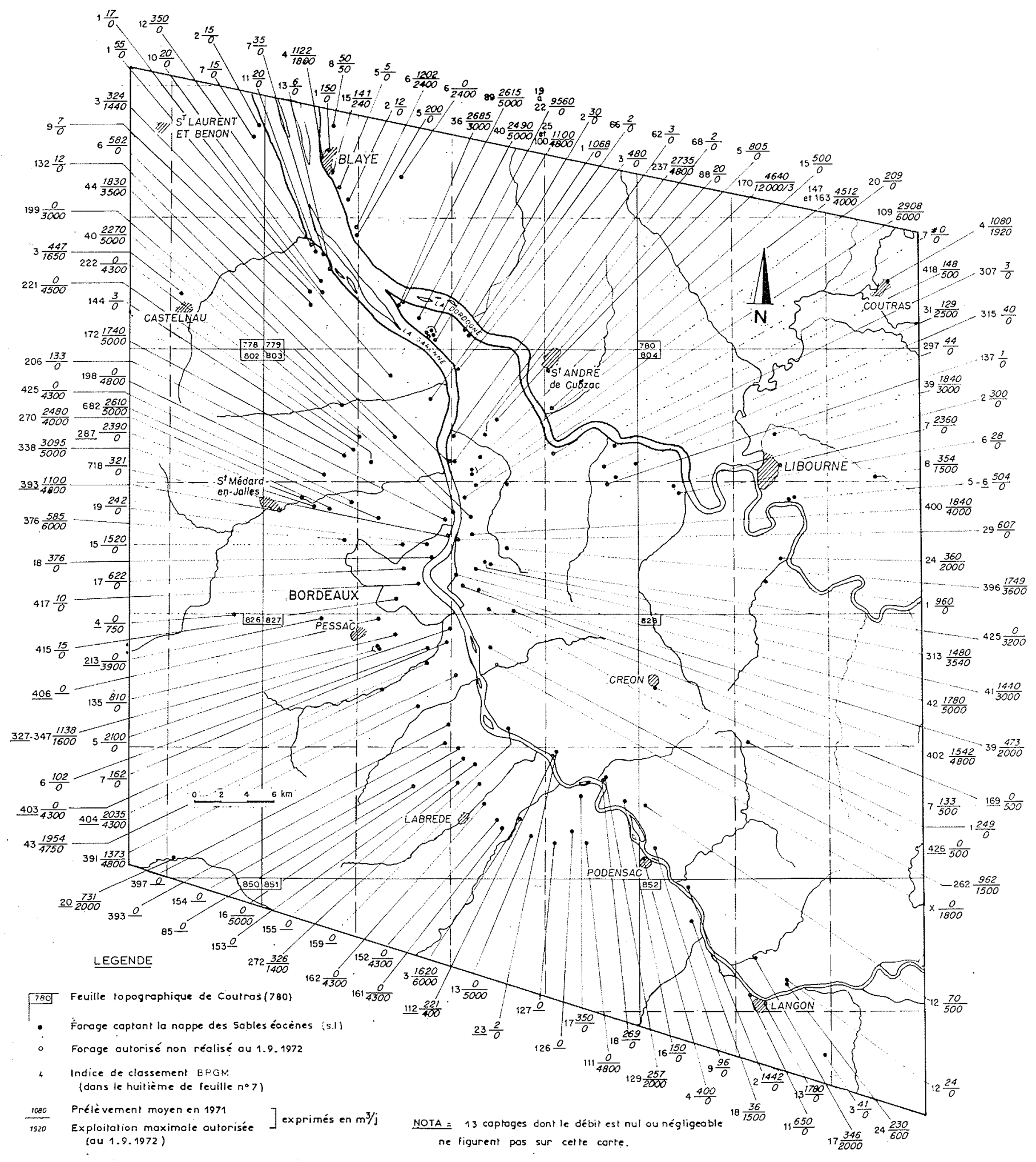

4/ Situation des points de prélèvements. 


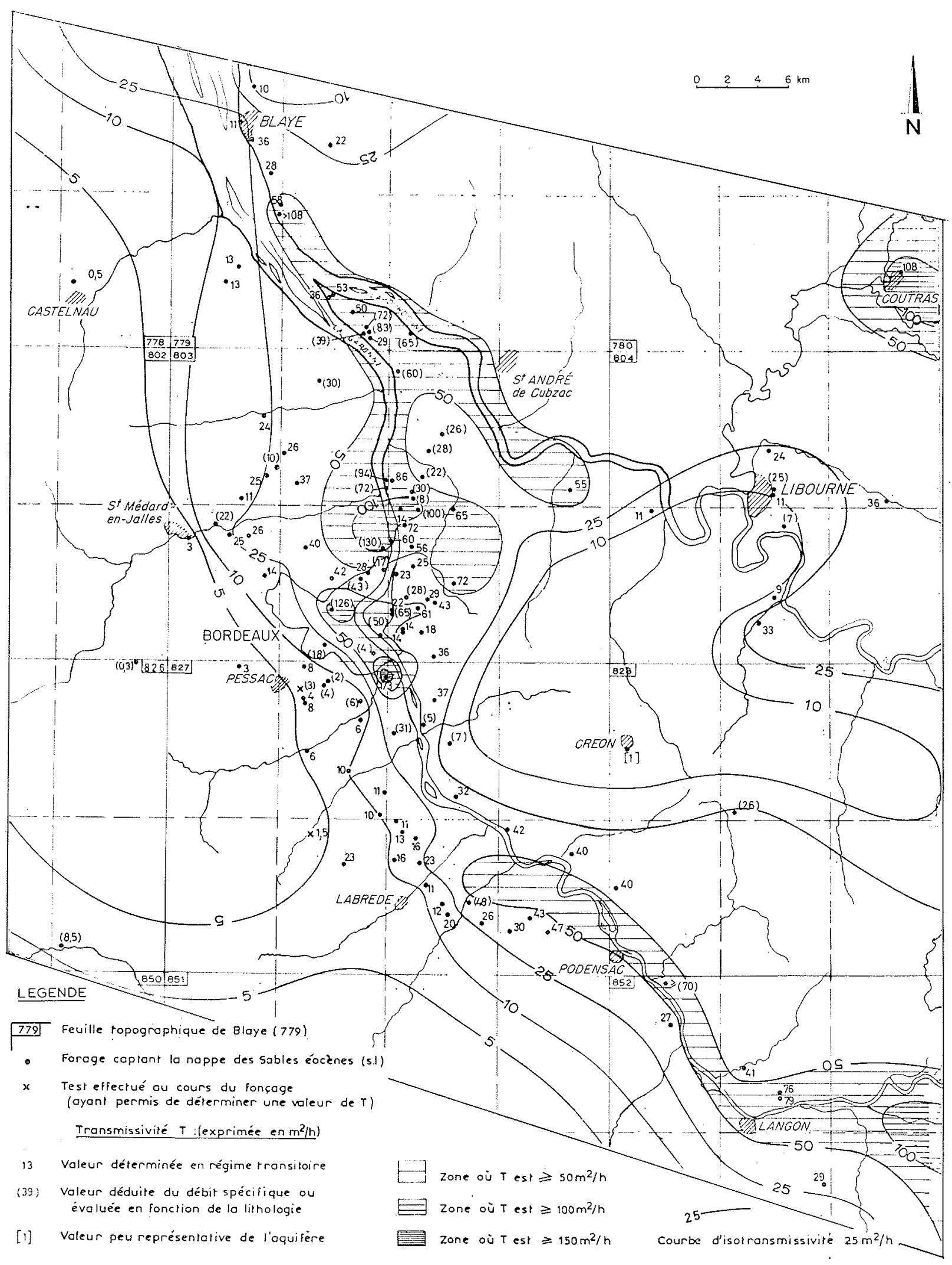

5/ Carte des transmissivités. 


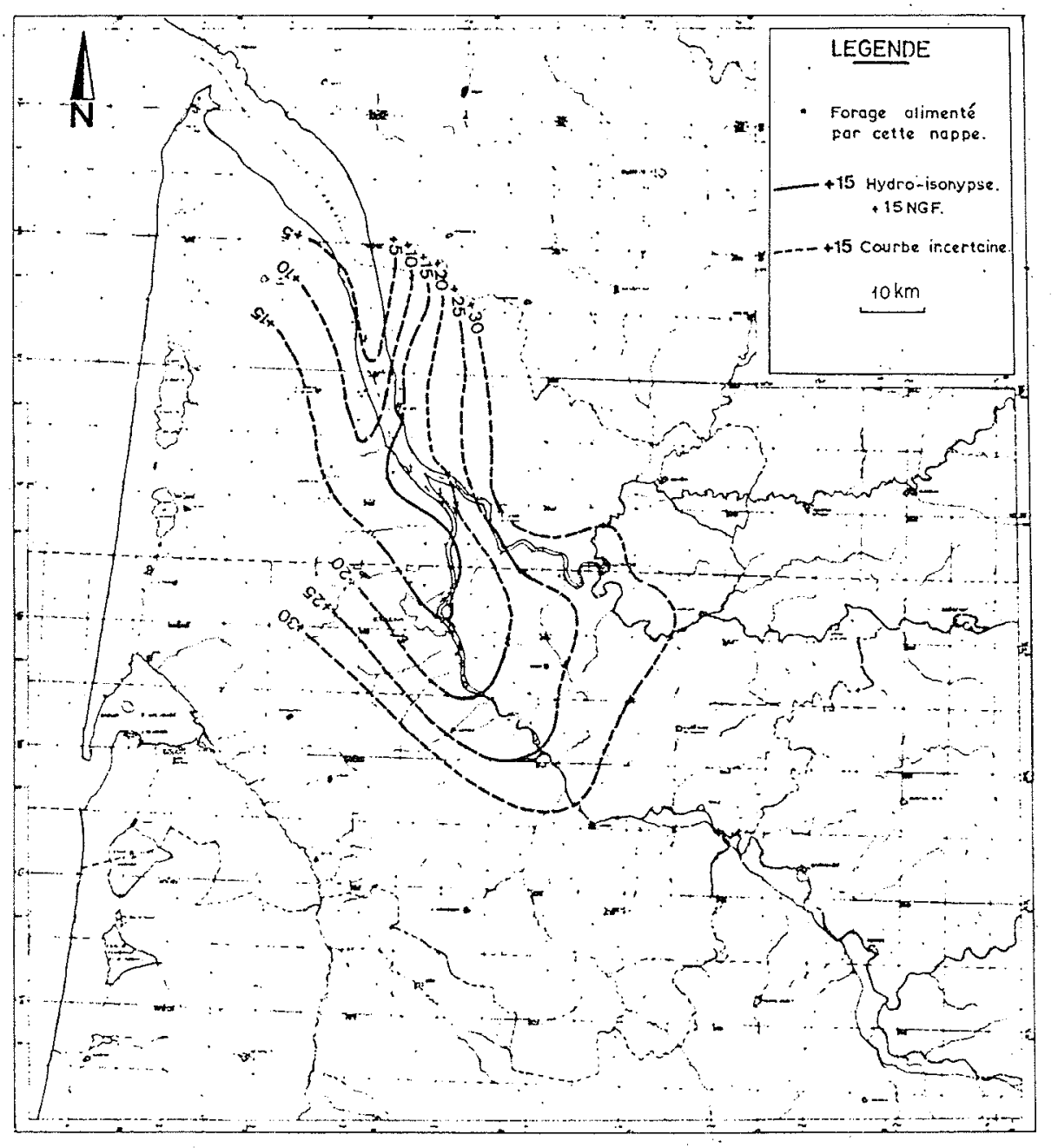

6/ Surface piézométrique, situation 1880-1890. 


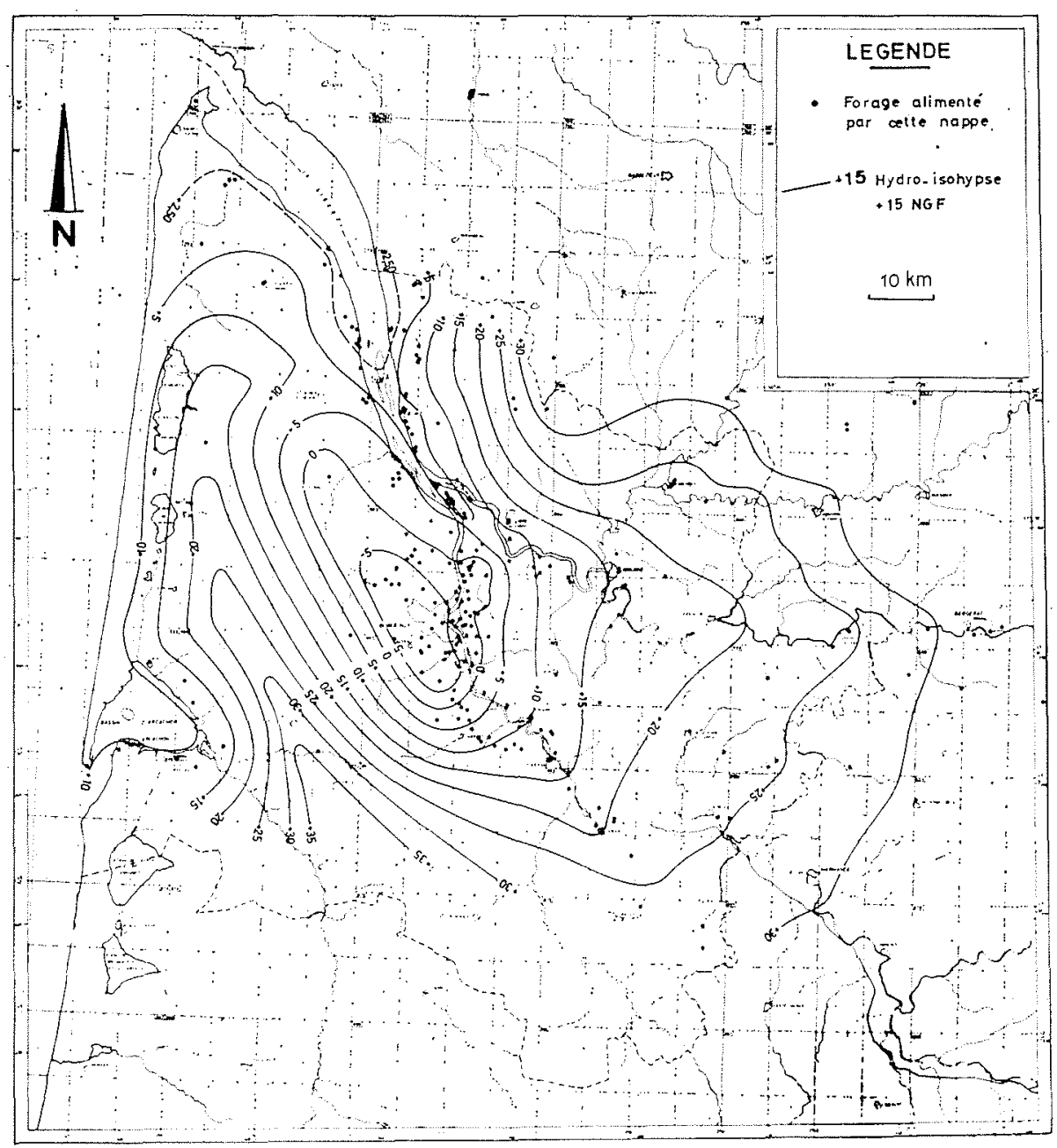

7/ Surface piézométrique, situation 1972. 


\section{Prélèvements}

En 1971, le cumul général des prélèvements se chiffre à $123000 \mathrm{~m}^{3} /$ jour pour le département de la Gironde et à $101600 \mathrm{~m}^{3} /$ jour pour le secteur étudié.

Ces prélèvements ont été regroupés en un certain nombre de secteurs. Pour les quatre principaux, on peut noter, en 1971, les prélèvements moyens suivants :

$\begin{array}{ll}\text { Secteur A : } 15600 \mathrm{~m}^{3} / \mathrm{j} & \text { Secteur C: } 46100 \mathrm{~m}^{3} / \mathrm{j} \\ \text { Secteur B : } 34200 \mathrm{~m}^{3} / \mathrm{j} & \text { Secteur D : } 8000 \mathrm{~m}^{3} / \mathrm{j}\end{array}$

Pour chacun de ces secteurs, la figure 9 indique l'évolution des prélèvements moyens cumulés pendant la période 1880 1971. Trois de ces diagrammes (A-B-C) mettent en évidence une très nette augmentation des pompages durant la dernière décennie, le dernier, inversement, montre une diminution de ceux-ci, liée à une réduction des débits spontanés d'anciens captages.

La vue de ces diagnammes est assez éloquente pour justifier et nos craintes et l'action entreprise.

\section{Les ressources en eau disponibles pour la recharge}

La quantité d'eau nécessaire annuellement pour atteindre les objectifs que nous nous étions fixés étant a priori inconnue en l'absence de simulation, il a été décidé de rechercher, dans la zone d'étude et à proximité immédiate, tous points d'eau superficielle ou de nappe phréatique capables de fournir ponctuellement un débit de 1001/s; ce débit pouvant paraître comme raisonnable pour chaque point d'injection.

Par ailleurs, excepté la Garonne et la Dordogne, rares sont les cours d'eau de la région qui ont un débit d'étiage régulier supérieur à ce chiffre. La recherche a même porté sur les nappes semi-profondes qui pouvaient fournir ponctuellement ce débit, ce qui pouvait faciliter un essai in sitit au moins dans un premier temps.

La qualité de ces eaux a également été abordée, ce qui nous a permis de nous rendre compte de l'extrême pauvreté de nos connaissances (surtout en ce qui concerne les eaux superficielles) et le manque de données relatives aux propriétés particulières permettant de juger de l'aptitude d'une eau à être injectée (pour les eaux souterraines notamment).

La partie de l'étude relative à la recherche des eaux injectables (quantité et qualité) doit être considérée comme une phase exploratoire au cours de laquelle le point a été fait sur la dooumentation existante.

Elle a surtout permis de mettre sur pied très tôt un cycle de campagnes de jaugeages et de prélèvements.

\section{Présélection et étude détaillée des zones favorables à la recharge}

Le premier problème que nous avons eu à résoudre a été d'établir la liste des critères et les valeurs limites dont on aurait à tenir compte pour déclarer favorable à la recharge telle ou telle zone.
Nous avons opéré par superposition des différentes cartes établies à l'occasion de l'étude géologique et hydrogéologique et, en particulier :

- de la carte des transmissivités et des débits spécifiques, en fixant comme limite $T>10 \mathrm{~m}^{2} / \mathrm{h}, Q S>5 \mathrm{~m}^{3} / \mathrm{h} / \mathrm{m}$;

- de la carte piézométrique, pour tenir compte des hauteurs de charge exploitables dans le cadre d'une injection gravitaire ( $25 \mathrm{~m}$ minimum);

- de la proximité des ressources potentielles pouvant être injectées;

- du périmètre de contrainte urbaine.

La synthèse de toutes ces contraintes aboutit à la localisation de trois zones A, B et C (fig. 10) que l'on peut considérer comme étant les plus favorables.

Pour chacune d'entre elles, une carte des isohypses du toit du réservoir le plus transmissif et une carte des isopaches des formations sableuses, ont été dressées à l'échelle du $1 / 50000^{\circ}$.

Ces cartes ne doivent pas faire illusion, car les informations ponctuelles que l'on possède sur ces zones sont très irrégulièrement réparties, sourvent imprécises (manque de diagraphies). De plus, on a pu se rendre compte, sur les coupes géologiques, du feuilletage des épontes du réservoir principal, des interconnexions avec les formations de

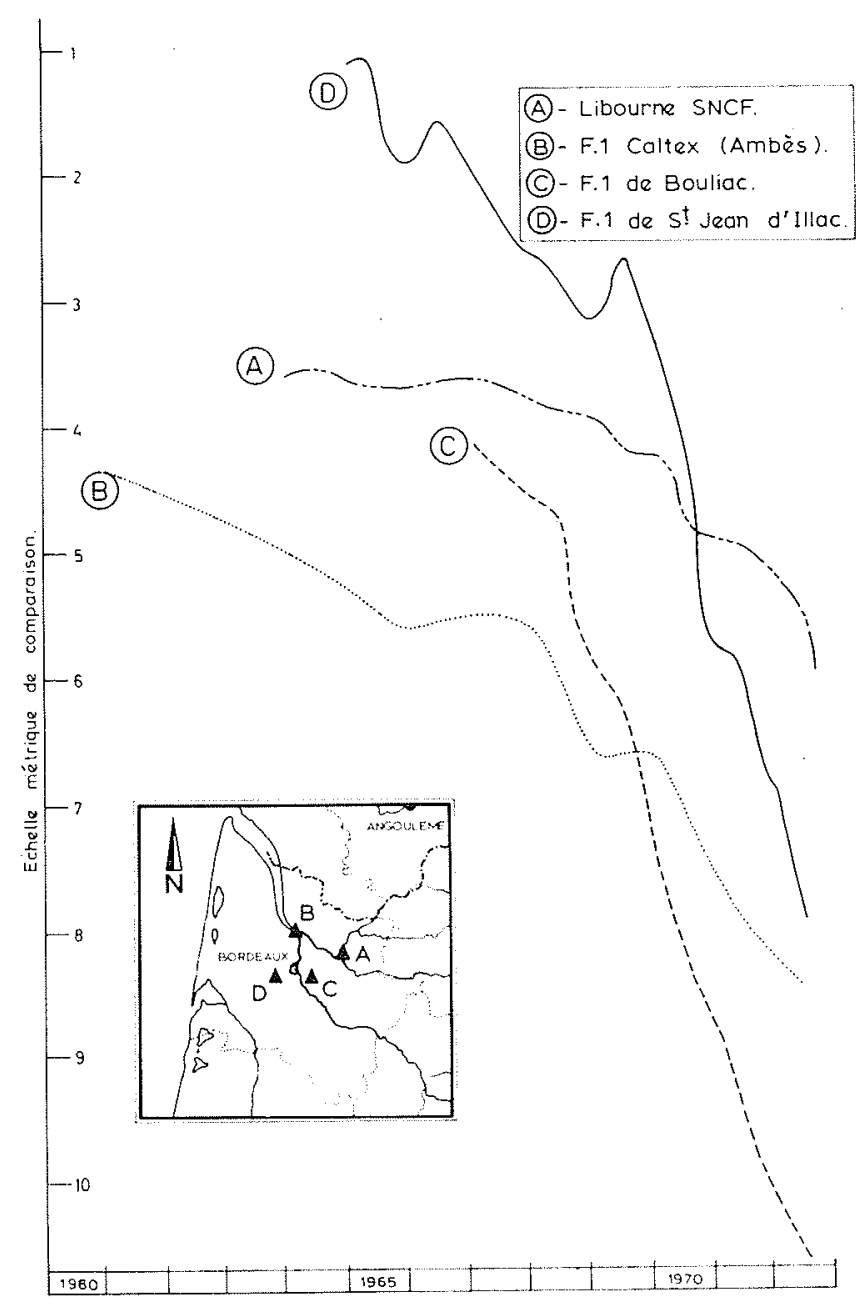

8/ Baisses piézométriques en quatre points distincts. 
l'Eocène supérieur et inférieur, voire de l'isolement d'une partie de l'aquifère. Dans ces conditions, il est très difficile de cartographier en isohypses (fig. 11) et en isopaches (fig. 12).

En ce qui concerne la zone A, que nous prendrons comme exemple, le secteur le plus favorable se situe entre Ludon au nord et Cachac au sud, le toit du réservoir se situe entre - 160 et $-200 \mathrm{NGF}$ environ, son épaisseur, assez constante, est de l'ordre de 50 à $65 \mathrm{~m}$ sous un faciès relativement homogène et la charge disponible se situe entre 10 et $40 \mathrm{~m}$, mais avec un grand nombre de forages au niveau desquels elle est comprise entre 20 et $30 \mathrm{~m}$.

Les principales ressources en eau disponibles pour la recharge sont, en ce qui concerne les eaux de surface, celles de la Jalle de Blanquefort et du ruisseau d'Artigue.

En ce qui concerne les eaux des nappes superficielles, les nappes desquelles on peut extraire des débits ponctuels de l'ordre de $100 \mathrm{~m}^{3} / \mathrm{h}$ sont trop hétérogènes pour qu'on puisse, sans étude détaillée, préciser les secteurs les plus favorables. Du point de vue chimique, ces eaux ont une teneur en fer souvent élevée; il serait nécessaire de mieux les connaitre de ce point de vue.

Les nappes semi-profondes du Miocène et de l'Oligocène ont de bonnes caractéristiques hydrauliques, possèdent une eau de qualité très comparable à celle de la nappe à réalimenter. Cependant, il n'est envisageable de les utiliser que pour des tests d'injection.

Dans la zone $A$, deux forages pourraient être utilisés en vue de l'injection dans une phase expérimentale : ils captent l'éocène moyen calcaire, puis sableux, grâce à des crépines de 8" de diamètre sur une centaine de mètres de hauteur, à des profondeurs allant de 150 à $250 \mathrm{~m}$, et à une colonne de captage de 13 " de diamètre. Les transmissivités sont de l'ordre de 26 à $37 \mathrm{~m}^{2} / \mathrm{h}$, les débits spécifiques vont de 11 à $15 \mathrm{~m}^{3} / \mathrm{h} / \mathrm{m}$.

\section{Simulation de l'alimentation artificielle}

Le modèle du bassin Nord-Aquitain a été adapté sur le programme SAMMIR (simulation des aquifères multicoutches à mailles irrégulières), mis au point par le Centro d'Informatique Géologique. Il permet la simulation, en régime permanent ou transitoire, des écoulements dans un aquifère multicouche suivant la loi de Darcy. L'espace est discrétisé en mailles carrées de dimensions variables. Le modèle comprend des couches perméables où l'écoulement
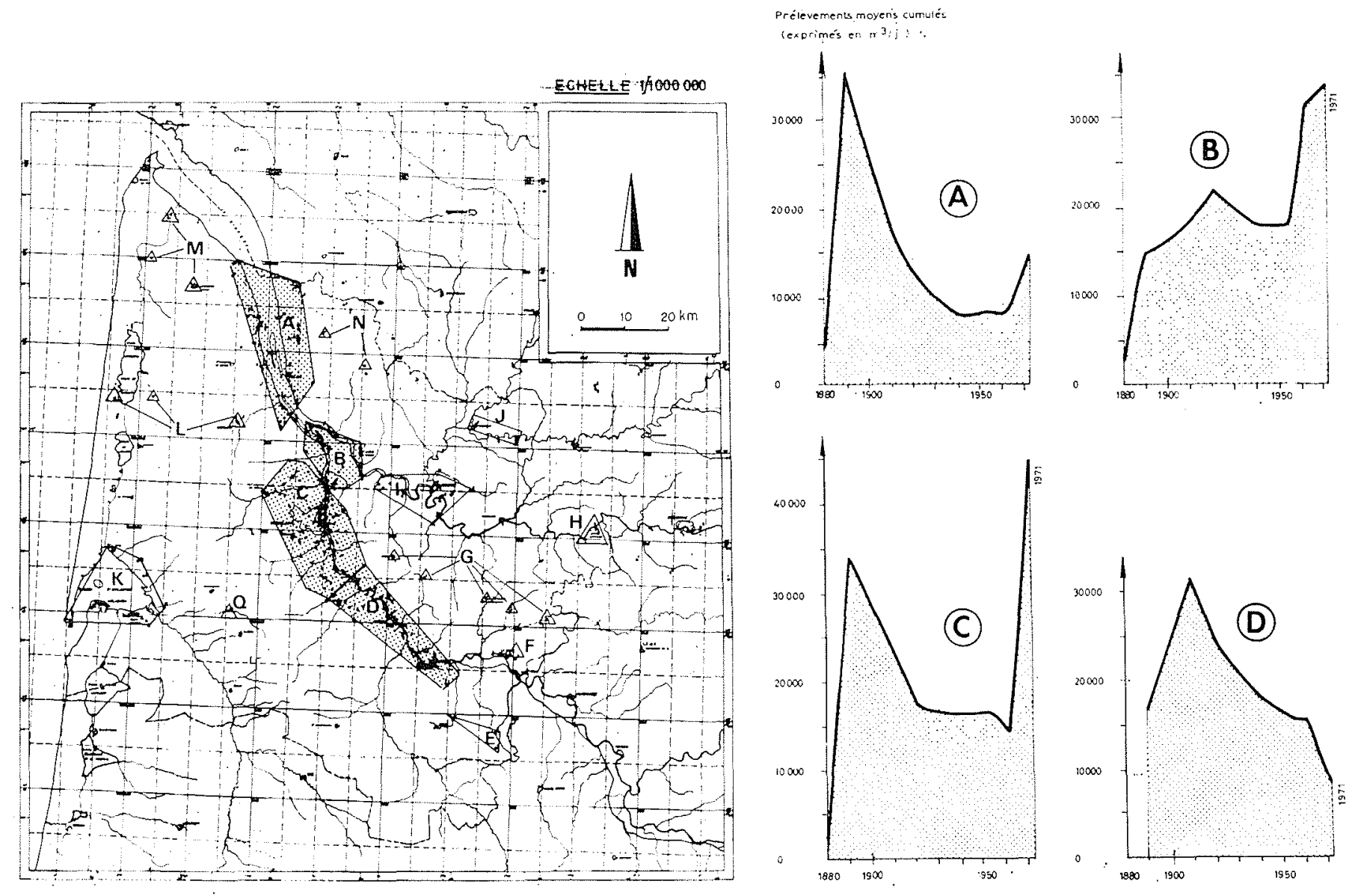

9/ Situation des zones de regroupement des prélèvements.

* Evolution graphique des prèlèvements moyens cumulés pour les quatre zones regroupant le plus grand nombre de captages (Période 1880-1971). 


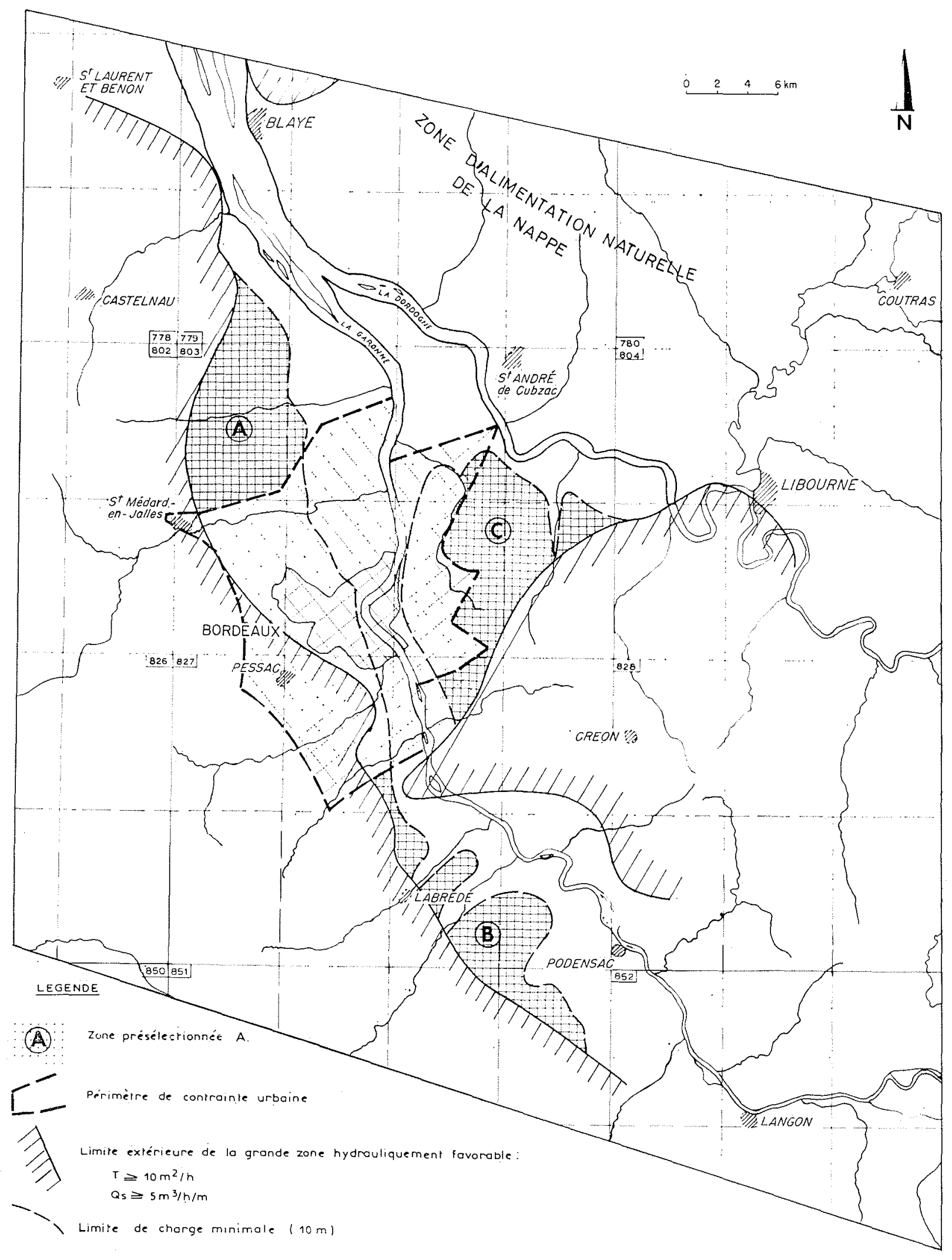

10/ Présélection des zones potentiellement favorables à la réinjection. 


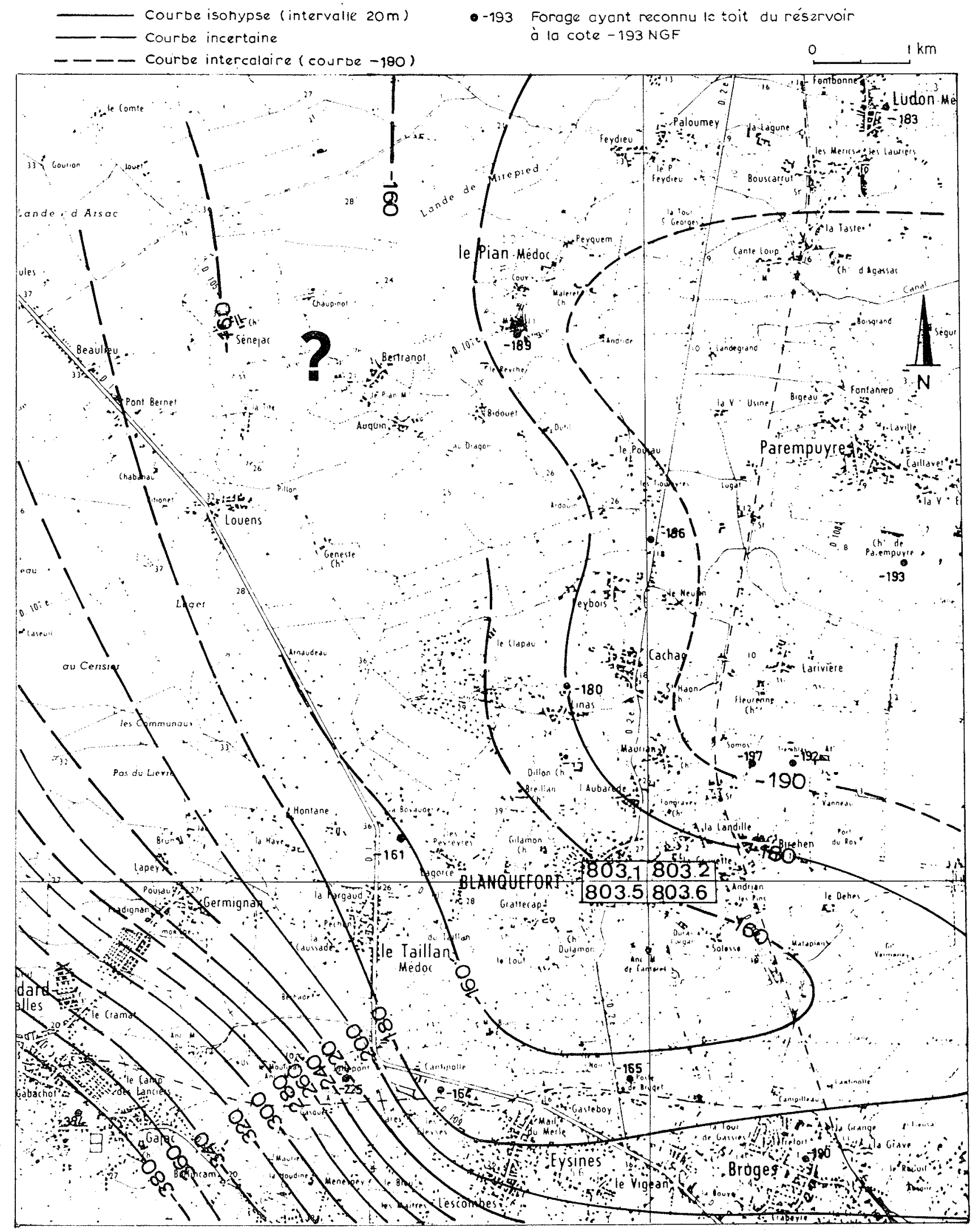

11/ Isohypses du toit du réservoir le plus transmissif. 


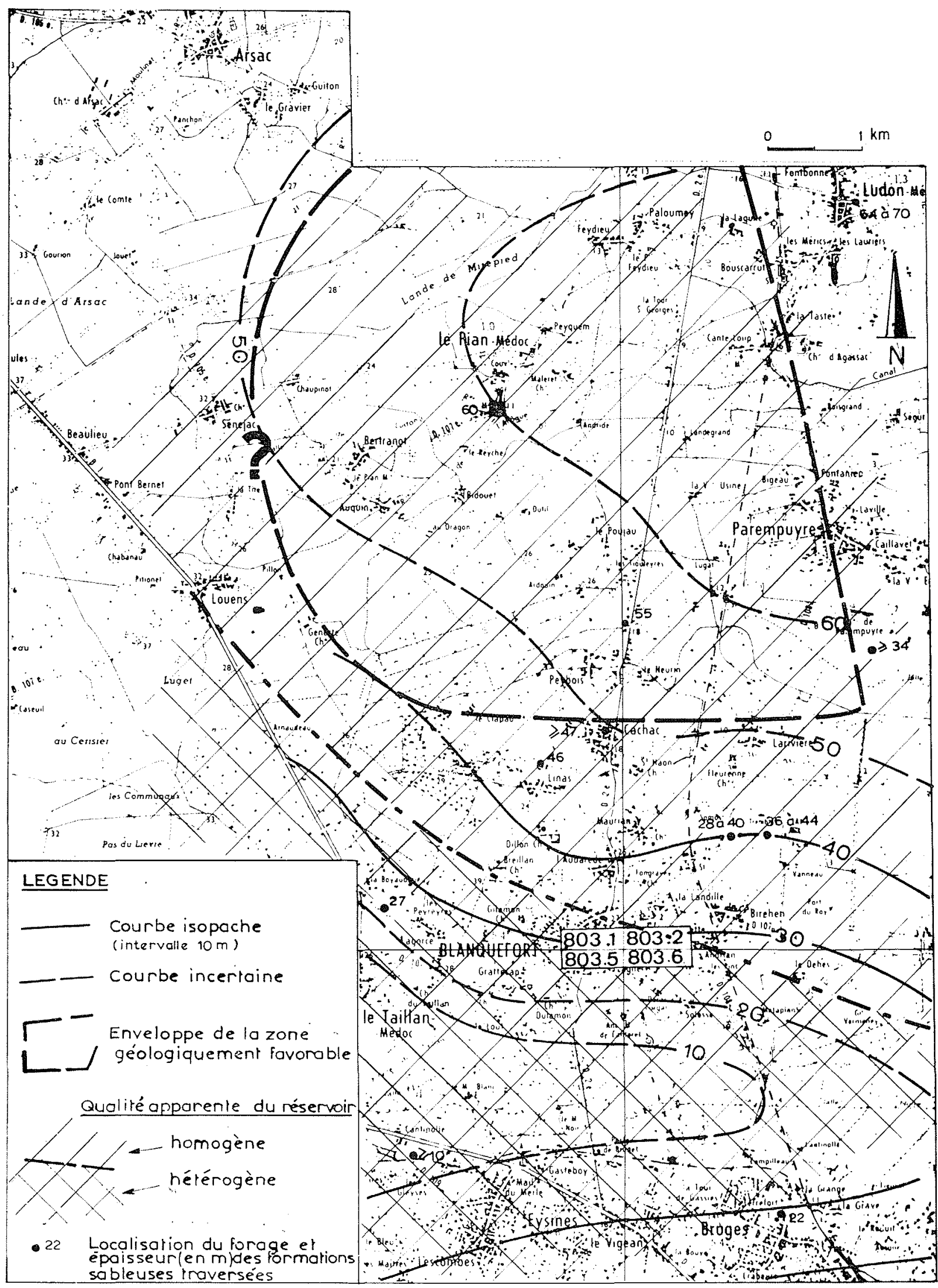

12/ Isopaches des formations sableuses. 
$13 /$

Etat de la nappe en 1972 Piézométrie restituée en régime transitoire.

14/ Zones favorables à la recharge retenues par le S.G.R.Aquitaine et mailles correspondantes où est simulée l'injection.

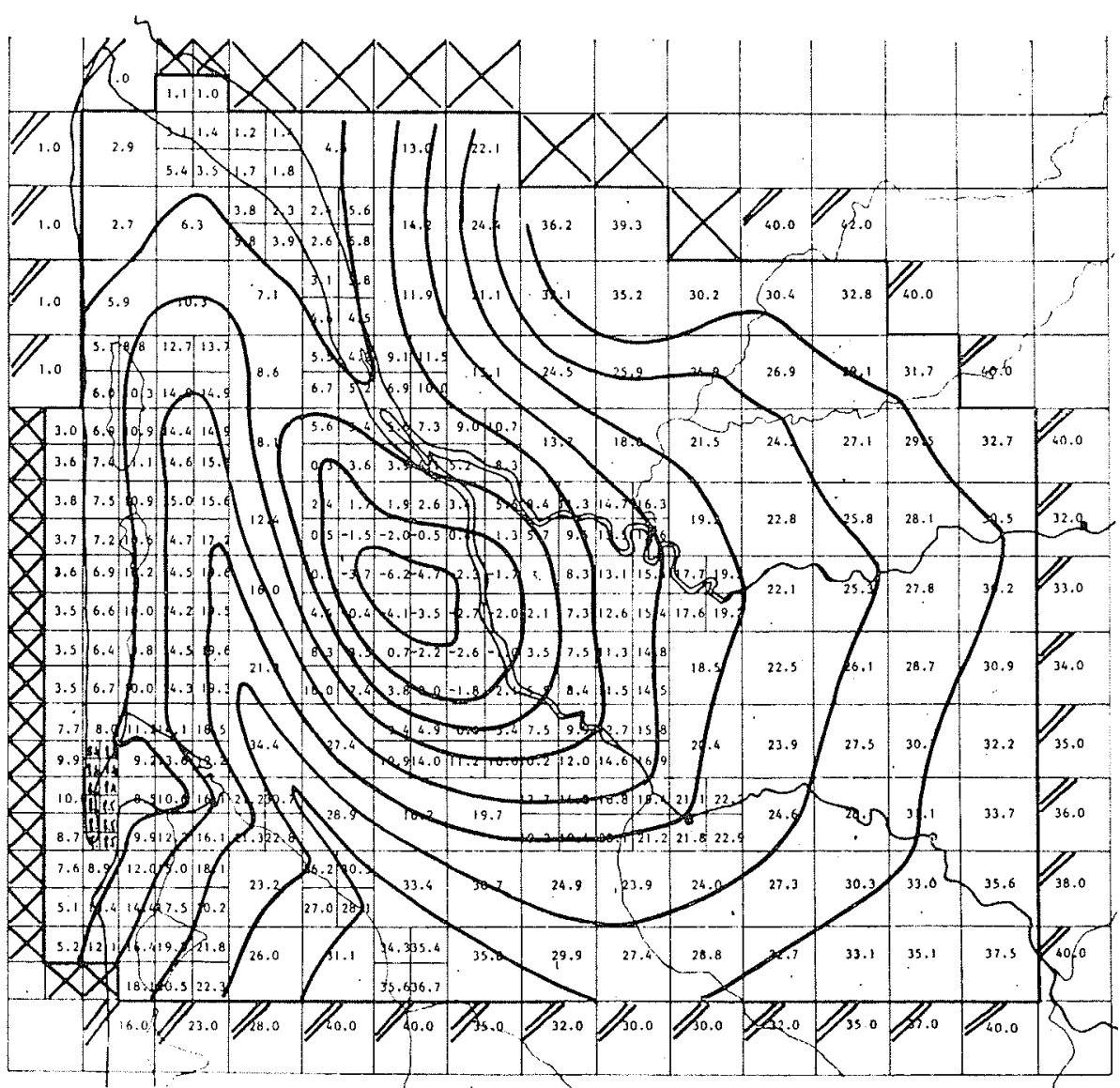

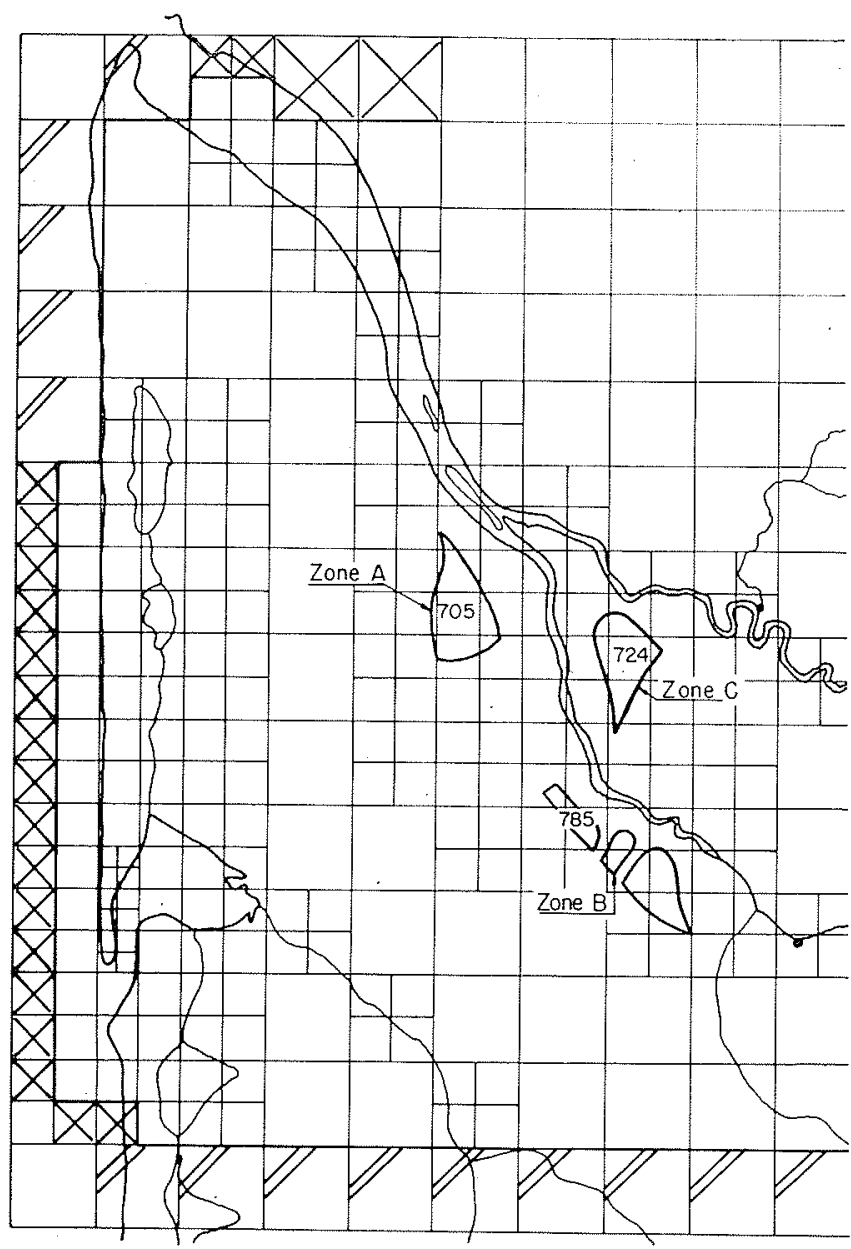

est bidimensionnel plan, séparées par des couches semiperméables à travers lesquelles s'établit une percolation verticale.

\section{Calage du modèle}

Le calage du modèle a été effectué d'abord par restitution de l'état piézométrique en 1965 , en prenant comme hypothèse de travail que cet état pouvait être assimilé à un régime permanent; puis en régime transitoire sur la période 1965-1972, comprenant trois phases de deux années chacune. La figure 13 présente la piézométrie ainsi obtenue, ce qui est une restitution très honorable compte tenu des hypothèses faites sur l'évolution des prélèvements entre 1965 et 1971, année pour laquelle nous possédions un relevé correspondant sensiblement à la réalité.

\section{Simulations réalisées}

La figure 14 montre la situation des trois zones présélectionnées par rapport au maillage du modèle.

\section{Injection d'un débit de $100 \mathrm{~m}^{3} / \mathrm{h}$}

Cette simulation a été réalisée successivement sur les trois zones, d'abord en régime permanent, puis en régime transitoire portant sur une période de quatre ans. On observera, par exemple, sur la zone A (fig. 15 et 16) les 


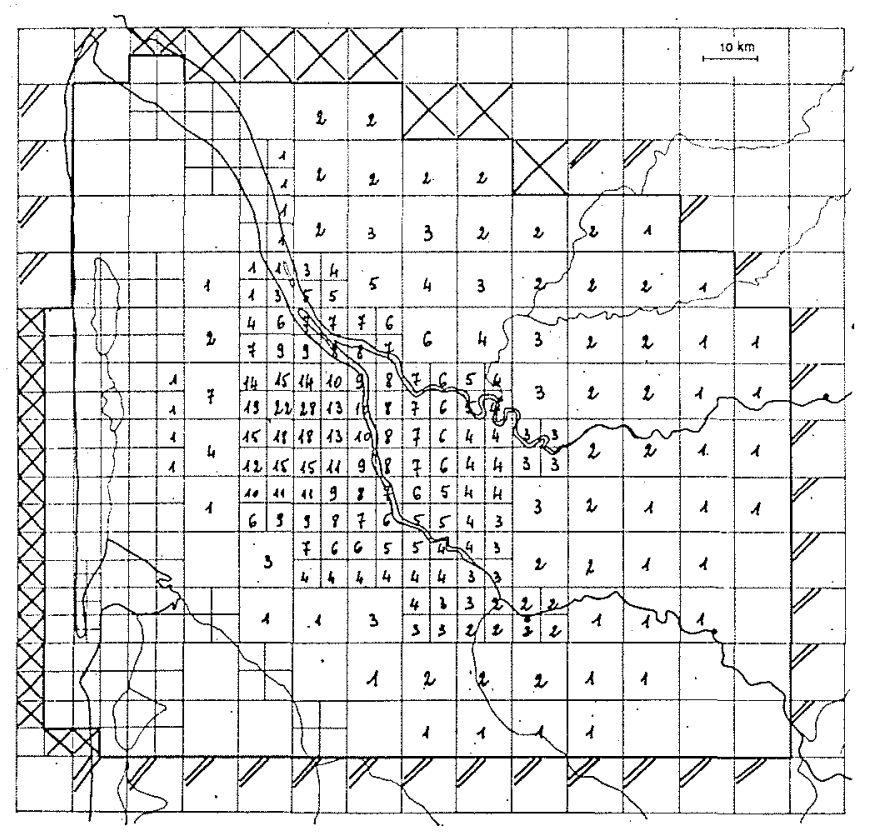

15/ Injection de $100 \mathrm{~m}^{3} / \mathrm{h}$ dans la maille 705 (régime permanent).

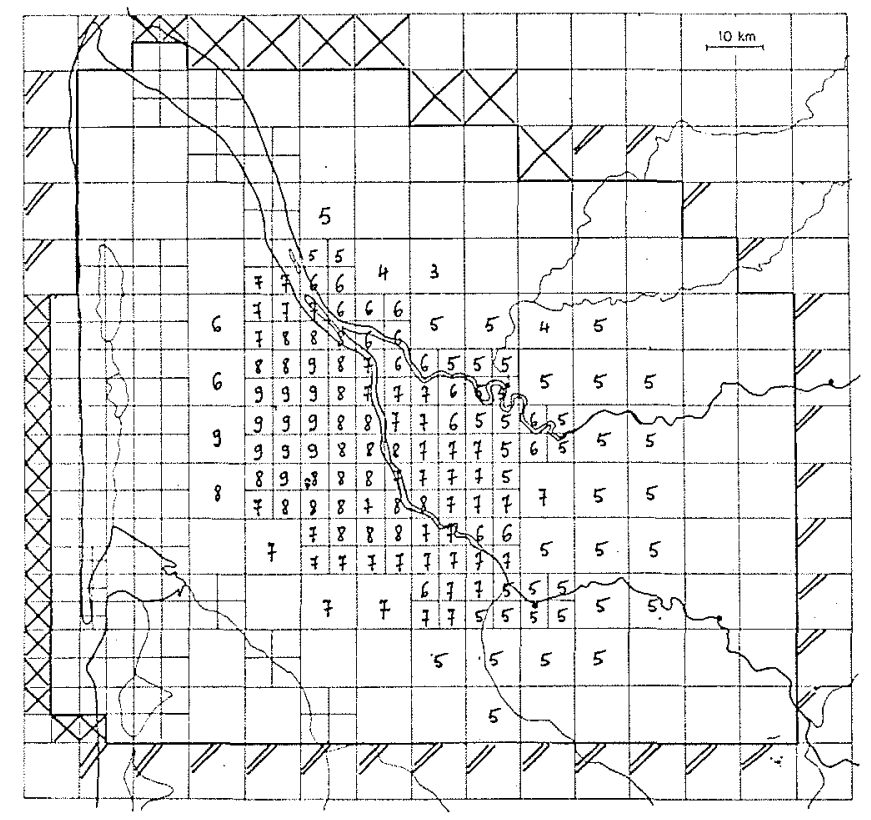

16/ Injection de $100 \mathrm{~m}^{3 / \mathrm{h}}$ dans la maille 705 (régime transitoire).

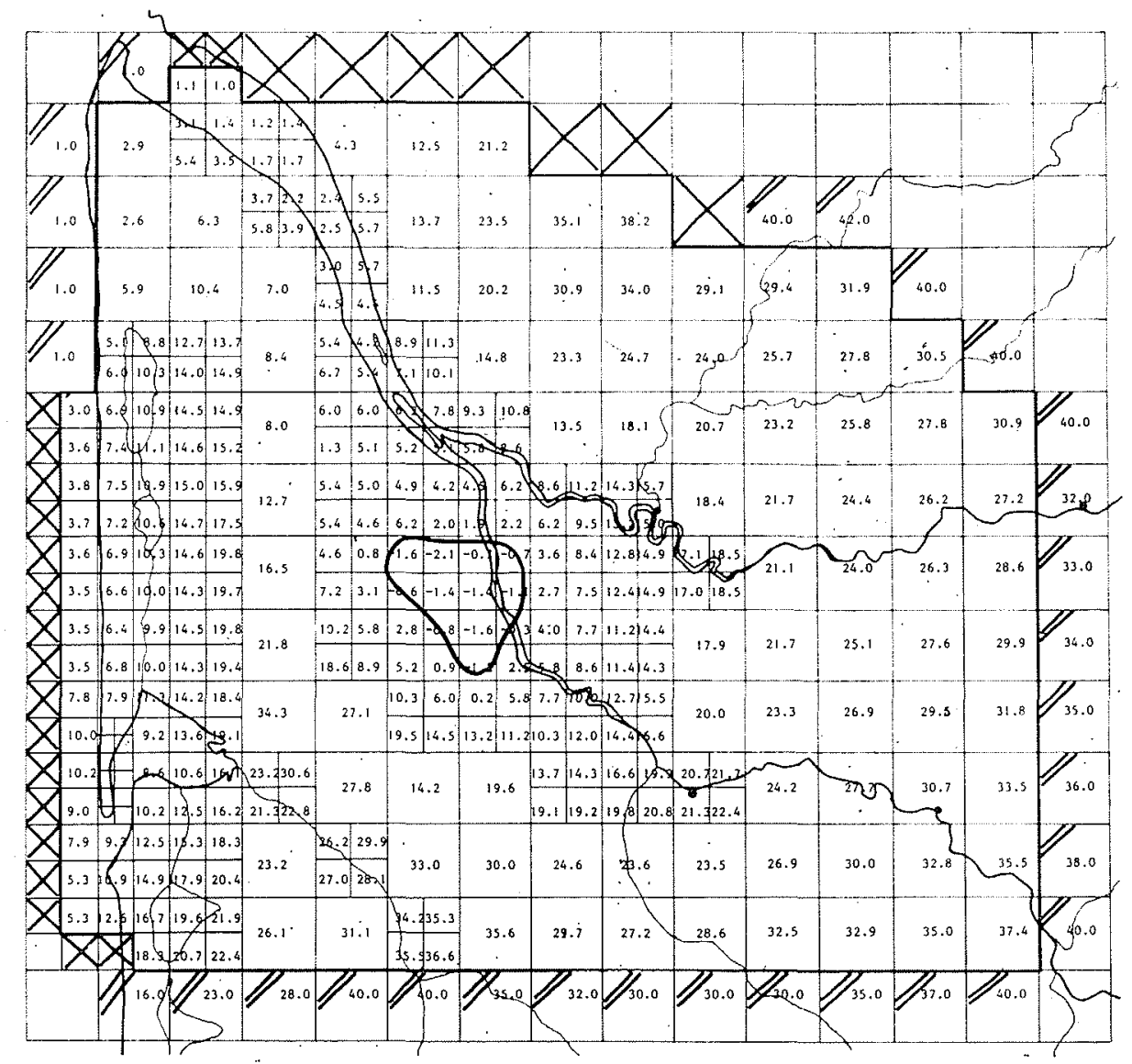

17/ Piézométrie calculée en permanent en simulant les prélèvements de l'année 1971 et l'injection de $360 \mathrm{~m}^{3} / \mathrm{h}$ de la maille 705 . 
valeurs de la remontée obtenue au niveau de chacune des mailles. Signalons que, par application du principe de superposition des écoulements, on peut envisager à volonté toutes les hypothèses d'injection à l'intérieur de ces trois mailles et obtenir les remontées correspondantes.

Les résultats obtenus en régime permanent et en régime transitoire ont été comparés. Nous constatons, qu'au voisinage de la maille $705,90 \%$ de la remontée obtenue en régime permanent sont atteints et parfois dépassés au bout de quatre ans d'injection, tous prélèvements maintenus constants par ailleurs. Sur une zone couvrant la totalité de la dépression, ce rapport s'établit à $80 \%$ pour la même période.

Il s'ensuit que l'on peut s'en tenir au régime permanent lors de prévisions du comportement de l'aquifère dans la zone déprimée. Il en résulte un gain de temps-machine dans un rapport de 1 à 5 .

\section{Débit à injecter pour supprimer toutes cotes négatives}

La simulation a été réalisée en régime permanent sur les trois zones considérées, en tenant compte des prélèvements en 1971.

Dans la zone A, par exemple (fig. 17), on constate qu'une injection de $360 \mathrm{~m}^{3} / \mathrm{h}$ ne suffit pas pour effacer le secteur à cotes négatives. Par application de ce qui a été dit précédemment, il convient d'injecter $100 \mathrm{~m}^{3} / \mathrm{h}$ supplémentaires pour obtenir le résultat escompté.

La comparaison des résultats obtenus sur les trois zones permet d'affirmer que la zone $\mathrm{A}$ a le meilleur rendement, puisqu'il faudrait respectivement 1000 et $800 \mathrm{~m}^{3} / \mathrm{h}$ supplémentaires pour atteindre le même résultat en injectant dans les zones $\mathrm{B}$ ou $\mathrm{C}$.

Par ailleurs, ces simulations font apparaître, dans certains cas, un gain en faveur des prélèvements lorsqu'on fait le rapport de l'augmentation des débits prélevés au débit à injecter. En toute logique, si l'état piézométrique final était strictement identique à l'état piézométrique initial, ce rapport devrait être égal à 1.
Or, il apparaît qu'un choix judicieux des points d'injection peut permettre d'obtenir des rendements supérieurs si l'on tend vers une piézométrie, non pas identique, mais équivalente, ce qui ne veut pas dire pour autant que cette dernière soit forcément plus défavorable. L'injection de $460 \mathrm{~m}^{3} / \mathrm{h}$ dans la zone A permet en effet d'obtenir un « rendement de recharge » égal à 1,45 .

\section{Prévisions de l'état de la nappe en $\mathbf{1 9 8 0}$}

Le calcul est fait en régime permanent. Il a été convenu d'adopter un taux de croissance des prélèvements de $5 \%$ par an, puis de supposer ces prélèvements constants pendant quatre ans pour tenir compte de la durée de mise en régime permanent du secteur intéressé par l'étude.

Par rapport à la situation actuelle, on obtient $10 \mathrm{~m}$ de dépression supplémentaire dans la zone centrale mais, ce qui est plus grave, des cotes négatives importantes (- 5,5 NGF) au bec d'Ambès, d'où un risque de contamination de l'Eocène à partir de cette région puisque, d'une part il affleure dans le lit de la Gironde et, d'autre part, il constitue localement le substratum d'alluvions flandriennes à eau salée.

\section{Etat piézométrique en 1980 avec alimentation artificielle}

Le calcul est fait en régime permanent dans le cas d'une injection de $1000 \mathrm{~m}^{3} / \mathrm{h}$ dans la maille 705 .

Par rapport à l'état actuel, le fond de la dépression est toujours à la même cote $(-6 \mathrm{~m})$. Mais le centre de cette dépression s'est déplacé vers le sud d'une vingtaine de kilomètres et une crête piézométrique sépare la dépression de la Gironde, ce qui constitue vis-à-vis d'une éventuelle pollution par la Gironde, un aspect positif.

Il faut se garder d'accorder une précision excessive aux résultats de ces simulations, cependant je pense que nous disposons d'un outil efficace pour nous guider sur la voie de la recherche d'une saine gestion de la nappe des sables éocènes. 


\section{Discussion}

Président : M. J. Margat

M. le Président remercie M. Plaud de son exposé et ouvre la discussion.

Comment, demande M. RocHE, compte-t-on amener l'eau de recharge au point d'injection à partir des ressources disponibles?

M. Plaud répond :

Pour l'instant, on en est à rechercher une eau en quantité suffisante mais aussi n'exigeant pas de frais excessifs de traitement avant injection, car pratiquement, il faut injecter dans les nappes une eau potable.

Dans tous les cas, l'amenée de l'eau aux points d'injection devrait se faire par conduite.

Pour le secteur nord-ouest de la nappe, je pense que l'on peut envisager l'exploitation de l'eau du Plioquaternaire du Médoc, ce qui permettrait, avec une seule adduction d'une vingtaine de kilomètres, de satisfaire les besoins en eau industrielle, au moins dans un premier temps, et les besoins dûs à l'alimentation artificielle de la nappe des sables éocènes.

Pour le secteur sud, la Communauté urbaine de Bordeaux envisage d'effectuer des prélèvements en Garonne pour faire face à l'accroissement prévisible des besoins en eau potable. Une station de traitement sera édifié; or, les stations de traitement fonctionnent rarement à pleine capacité. Celle-ci pourrait, en période creuse, produire l'eau destinée à être injectée, ainsi les investissements seraient rentabilisés au maximum.

Pourriez-vous nous donner une idée des techniques d'injection, demande M. Roche?

La communication suivante due à mon collègue, M. Bize, vous renseignera sur l'alimentation artificielle des sables de l'Albien dans le bassin de Paris, dit M. Plaud. A Bordeaux, on pratiquera sensiblement de la même façon; le forage fonctionne à l'envers du schéma classique.

M. Thirriog pose les deux questions ci-après :

$1^{\circ}$ Pourriez-vous nous donner l'explication du rendement de $150 \%$ de la réalimentation dont vous avez parlé au cours de votre exposé ?

$2^{\circ}$ Dans le cadre de la région intéressée, on peut se demander si la réalimentation répond à un objectif économique ou à des nécessités psycho!ogiques on politiques?

Est-ce que, pour ne pas changer les habitudes des Bordelais, on injecte de l'eau potable en un certain endroit et on la récupère 10 ou $20 \mathrm{~km}$ plus loin, le milien poreux servant de réseau de distribution

Est-il moins cher d'utiliser le sous-sol comme moyen de transport au lieu du transport classique par conduites ou canaux.
Pourriez-vous nous donner un ordre de grandeur des conts respectifs de ces deux solutions?

A la première question, M. Plaud répond qu'à la réflexion, l'emploi du terme rendement, très séduisant a priori, s'avère en effet, quelque peu inadapté.

L'hypothèse de travail, faite en début d'expérimentation, consistait à considérer la situation piézométrique de 1965 comme un état de pseudo-équilibre, les prélèvements représentaient alors $1135 \mathrm{l} / \mathrm{s}$. En 1971, ils étaient de $13201 / \mathrm{s}$, mais l'injection de $128 \mathrm{l} / \mathrm{s}$, simulée sur le modèle, faisait apparaître une piézométrie équivalente à celle de 1965

L'injection revient alors à une limitation de prélèvements à $1320-128=1192 \mathrm{l} / \mathrm{s}$, pour lesquels on estime qu'un état de pseudo-équilibre peut être atteint.

Etant donné les difficultés à comparer deux états piézométriques lorsqu'ils ne sont pas strictements identiques, on peut considérer que le supplément de ressources exploité, de $1135 \mathrm{l} / \mathrm{s}$ à $11921 / \mathrm{s}$, soit $571 / \mathrm{s}$, représente, en terme de débit, la différence entre les deux états piézométriques, l'état final étant à une cote moyenne inférieure à celle de l'état de 1965.

Quoi qu'il en soit, ce qu'il faut apprécier c'est le rétablissement d'un état piézométrique acceptable eu égard aux risques encourus, accompagné d'un supplément de ressources obtenu sur des puits hors de la zone la plus déprimée et qui a peu d'influence sur son comportement,

La réponse à là deuxième question nécessite d'avoir connaissance de la situation. Il existe, à Bordeaux, une nappe techniquement mal exploitée par environ deux cents forages, ce qui représente des investissements importants. Ceux-ci risquent de perdre tout ou partie de leur valeur du fait de l'abaissement continu du niveau de la nappe et des risques d'invasion par l'eau salée. Il y a, par ailleurs, des besoins domestiques et industriels qui entrent en compétition. Il paraît souhaitable de réserver les eaux souterraines aux usages domestiques et d'alimenter en eau industrielle... les industries; surtout lorsque l'on sait que les ressources de cette nappe ne pourront plus, à terme, satisfaire les besoins des uns ou des autres.

La réalimentation, assortie des mesures que j'ai énnoncées, répond donc à un objectif économique réel, tout en préservant les aspirations légitimes des Bordelais.

Pour ce qui concerne le coût de la réalimentation et sa comparaison avec ceux d'adductions classiques, il convient d'attendre le résultat du dernier volet de l'étude, mais il faudra se garder de comparer brutalement des chiffres, car la réalimentation comporte, sur l'adduction classique, des avantages importants difficilement quantifiables.

M. le Président clôt la discussion et donne la parole à M. BIZE pour l'exposé de sa communication. 